\title{
Comparison of Buttock Pressure and Pelvic Tilting Angle During Typing in Subjects With and Without Unilateral Low Back Pain
}

\author{
Ui-jae Hwang ${ }^{1,2}$, BHSc, PT, Si-hyun Kim ${ }^{1,2}$, MSc, PT, Houng-sik Choi ${ }^{3}$, PhD, PT, \\ Oh-yun Kwon ${ }^{4}, \mathrm{PhD}, \mathrm{PT}$ \\ ${ }^{1}$ Dept. of Physical Therapy, The Graduate School, Yonsei University \\ ${ }^{2}$ Kinetic Ergocise Based on Movement Analysis Laboratory \\ ${ }^{3}$ Dept. of Physical Therapy, Division of Health Science, Hanseo University \\ ${ }^{4}$ Dept. of Physical Therapy, College of Health Science, Yonsei University
}

\begin{abstract}
Asymmetric sitting posture may cause asymmetric buttock pressure and unilateral low back pain (LBP). The purpose of this study was to compare the differences of buttock pressure between both sides, and pelvic angle (sagittal and coronal planes) during typing in a sitting position on a pressure mat (Baltube) in individuals with and without unilateral LBP. Ten subjects with unilateral LBP and ten subjects without unilateral LBP were recruited for this study. Buttock pressure was measured using a pressure mat and pelvic angles were measured using a palpation meter. The subjects performed typing in a sitting posture for 30 minutes. Pressure data were collected and averaged at initial term (from start to first minutes) and final term (last minutes of 30 minutes). Angles of pelvic tilting were measured after 30 minutes typing. Pressure asymmetry values (difference in pressure between both sides) were calculated at the initial and final terms. A two-way analysis of variance was used to compare the differences between the initial and final pressure asymmetry values in subjects with and without unilateral LBP. An independent $\mathrm{t}$-test was applied to compare the pelvic tilt angles between the two groups. To compare the change of pressure from the initial term to the final term between the symptomatic and asymptomatic sides in the unilateral LBP group, a paired t-test was applied. In the unilateral LBP group, the pressure asymmetric value at the final term was significantly greater than that of the initial term $(p<.05)$. The angle of pelvic tilting in coronal plane was significantly greater in the unilateral back pain group compared to the without unilateral LBP group $(\mathrm{p}<.05)$, however, there was no significant difference in the angle of pelvic tilting in the sagittal plane between the two groups ( $>.05)$. In the unilateral LBP group, the change of pressure from the initial term to the final term was significantly less in the symptomatic side $(-6.90 \mathrm{mmHg})$ than the asymptomatic side $(5.10 \mathrm{mmHg})$. This asymmetric sitting posture may contribute to unilateral LBP in the sitting position. Further studies are needed to determine if asymmetric weight bearing in sitting causes unilateral LBP or if unilateral back pain causes asymmetric weight bearing, and if the correction of asymmetric weight bearing in sitting can reduce unilateral LBP.
\end{abstract}

Key Words: Buttock pressure; Pelvic tilting angle; Sitting posture; Typing; Unilateral low back pain.

\section{Introduction}

The prevalence and incidence of musculoskeletal disorders have increased in computer workers (Aarås et al, 1997; Watanabe et al, 2007). Studies conducted by the National Institute for Occupational Safety and Health proved that $20.25 \%$ of 1,000 computer workers felt continuous discomfort in the back area (Sauter et al, 1991). Low back pain (LBP) has been linked to asymmetrical sitting posture and pelvic positions

Corresponding author: Oh-yun Kwon kwonoy@yonsei.ac.kr 
(Al-Eisa et al, 2004; Grundy and Roberts, 1984; Knutson 2002). Pelvic asymmetry is a commonly reported aggravating factor for LBP (Dankaerts et al, 2006; Vergara and Page, 2002; Williams et al, 1991; Womersley and May, 2006) and sitting position has been considered as individual sitting posture (Dankaerts et al, 2006). It is a commonly viewed that pelvic asymmetry contributes to secondary altered alignment in the lumbar spine, like scoliosis (Riegger-Krugh and Keysor, 1996). Altered alignment shows as a lateral curve that involves various degrees of vertebral rotations as well. Commonly, it is this secondary alterations that are assumed to lead to LBP and contribute to secondary structural alterations in the spine (McCaw and Bates, 1991). Pelvic asymmetry is generally related to sacroiliac joint dysfunction, and abnormal alignment between the right and left innominate bones on the sagittal plane, specifically, iliac rotation asymmetry (Egan et al, 1996).

Pelvic asymmetry also causes kinematic changes in the spine and may affect vertebral disks. Nachemson (1975) studied the disparity in the compressive force on the disks in various positions. Compression influences the disks, the facet joints and alignments. The effect of compression is not concrete to any diagnosis or category but should be considered a factor in the impairment movement of LBP patients.

Lee and Yoo (2011) measured the pressure between the seat and the gluteus using the Tekscan system (Tekscan Inc., South Boston, MA, USA) to investigate the change of gluteal pressure by crossed leg sitting position, and they reported that crossed leg sitting causes asymmetric buttock pressure. In other studies, Park and Yoo (2011) administered pressure biofeedback to frequent computer users using a posture-sensing air seat device for monitoring trunk movements and they reported that a posture-sensing air seat device can reduce trunk and lateral flexion. But this study did not directly measure buttock pressure and pelvic angles. There was comparison of erector spinae muscle volumes at the L5-S1 levels and multifidus muscle volumes at the L4 and S2-3 levels of cross sectional area with and without unilateral low back pain (Beneck and Kulig, 2012) and they reported that the LBP group had an $18.1 \%$ reduction in muscle volume at the $\mathrm{L}^{-}{ }^{-} \mathrm{S} 1 \mathrm{lev}^{-}$ els, but no pain and non-pain side interaction effect was identified.

Previous studies that measured buttock pressure in the sitting position used Tekscan in normal healthy subjects (Lee and Yoo, 2011). Although previous literature postulated that asymmetric sitting posture may contribute to unilateral back pain (Park and Yoo, 2011), there have been no studies that have investigated asymmetric weight bearing in individuals with unilateral LBP.

The purposes of this study were (1) to compare differences of buttock pressure between both sides in subjects with and without unilateral LBP, (2) to compare the pelvic tilting angles in subjects with and without unilateral LBP after 30 minutes of computer typing, and (3) to compare the change of buttock pressure from the initial term to the final term between the symptomatic and asymptomatic sides in subjects with unilateral LBP. It was hypothesized that the unilateral LBP group would demonstrate greater asymmetric buttock pressure and pelvic tilting angle compared to the without LBP group. It was also hypothesized that the change of buttock pressure in the symptomatic side would be less than in the asymptomatic side in subject with unilateral LBP.

\section{Methods}

\section{Subjects}

Sample size was calculated in this study for G*power analysis. By utilizing G*power 3.1.2 software (Franz Faul, University of Kiel, Kiel, Germany) in a pilot study of 16 subjects, an effect size of 4 was confirmed (calculated by partial $n^{2}$ of .78 at an 
Table 1. Characteristics of the subjects

$(\mathrm{N}=20)$

\begin{tabular}{ccc}
\hline \hline Variables & $\begin{array}{c}\text { Unilateral LBP group } \\
\left(\mathrm{n}_{1}=10\right)\end{array}$ & $\begin{array}{c}\text { Without unilateral LBP group } \\
\left(\mathrm{n}_{2}=10\right)\end{array}$ \\
\hline Age (year) & $22.7 \pm 3.43^{\mathrm{a}}$ & $22.7 \pm 1.6$ \\
Weight $(\mathrm{kg})$ & $64.0 \pm 13.4$ & $63.1 \pm 6.9$ \\
Height $(\mathrm{cm})$ & $173.8 \pm 8.2$ & $172.4 \pm 5.5$ \\
VAS $^{\mathrm{b}}(\mathrm{mm})$ & $55.0 \pm .7$ & \\
Duration of unilateral LBP $^{\mathrm{c}}$ (month) & $8.8 \pm 3.8$ & \\
MODI $^{\mathrm{d}}(\%)$ & $17.6 \pm 6.4$ & \\
\hline
\end{tabular}

${ }^{a}$ mean \pm standard deviation, ${ }^{b}$ visual analogue scale, ${ }^{c}$ low back pain, ${ }^{d}$ modified Oswestry disability index.

alpha level of .05 and a power .80) (Faul et al, 2007).

Twenty subjects (13 males and 7 females) were recruited among Yonsei University students. The unilateral LBP group consisted of ten (6 males and 4 females). These included nine subjects of right side pain and one subject of left side pain. The characteristics of subjects are listed in Table 1. The inclusion criteria for the unilateral LBP group were as follows: 1) pain was consistently unilateral and lo- $^{-}$ calized in the low back region 2) the first onset of LBP must have occurred at least 1 year before testing 3) the subject must have had at least 1 episode of LBP within the last 6 months 4) symptoms were minimal or absent for at least 3 days before testing (Beneck, 2012). The exclusion criteria for the unilateral LBP group were as follows: 1) bilateral leg symptoms 2) prior low back surgery 3) structural scoliosis or spondylolisthesis 4) pregnancy 5) the subject identified that he or she might limit participation in physical activity. The without unilateral LBP group consisted of ten subjects without unilateral LBP (7 males and 3 females) that had no experience of unilateral LBP, and didn't undertake postural control exercise. Before the study, the investigator explained all of the method and procedures to the subjects.

To measure the severity and perception of LBP, a visual analogue scale (VAS) and a modified Oswestry disability index (MODI) were used. The severity and perception of the unilateral LBP experience were measured using the VAS (Marshall and Murphy, 2010). The VAS consisted of a $10 \mathrm{~cm}$ line with "no pain" labeled as 0 and "worst pain" as 10 . Subjects with unilateral LBP were asked to check their pain degree on the line. The Oswestry Disability Index (ODI) was used to measure pain and disability in LBP patients (Marshall and Murphy, 2010; Maughan and Lewis, 2010). The MODI consists of 10 items with six answers per item, and those were scored from 0 to 5 . The total score was shown as an associative value (total possible score 9/total score 100) for participants.

\section{Instruments}

\section{Pressure mat}

Pressure mat (Baltube, RELIVE, Gimhae, Korea) is a pressure measurement device used while sitting. The size of the mat is $25 \mathrm{~cm} \times 25 \mathrm{~cm} \times 7 \mathrm{~cm}$, and it is divided into four air chambers. The first chamber is the right buttock side, the second chamber is the right thigh side, the third chamber is the left buttock

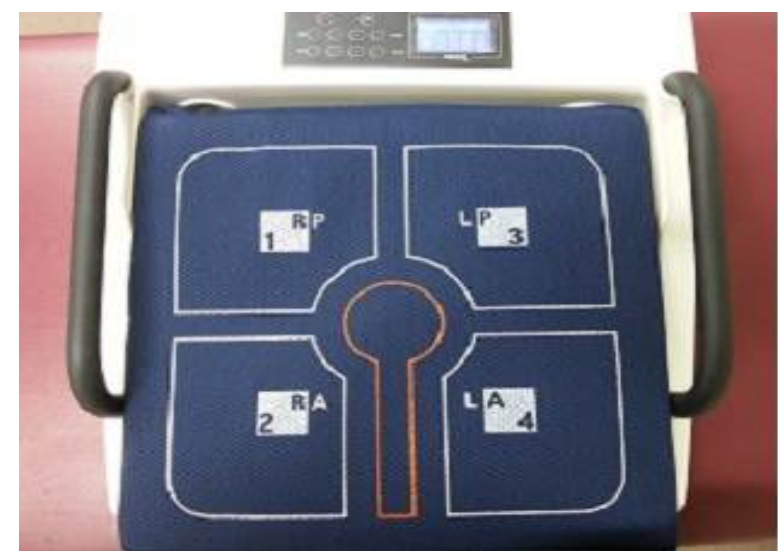

Figure 1. Pressure mat. 
side, and the fourth chamber is the left thigh side. Figure 1 shows the position of the air chambers, with the right and left sides, and the anterior and posterior sides, being labeled as R, L, A, and P, respectively. The subjects were instructed to sit on the correct chamber with both the buttocks and the thighs. The four chambers measured the pressure from the buttocks and the thighs. Their sensors could accommodate pressure until a maximum of 999 $\mathrm{mmHg}$. If pressure passes $150 \mathrm{mmHg}$, the sensors switch off automatically to protect the seat. The sensors in Baltube seats are silicon pressure sensors with series-standard accuracy (TruStability $\left.{ }^{\circledR}\right)$. Silicon pressure sensors estimate a ratiometric analog output for scanning pressure value over the special full scale pressure span.

\section{Palpation meter}

The palpation meter (PALM, Performance Attainment Associates, USA) was developed to measure pelvic angle in the sagittal and coronal planes (Figure 2). Intraclass correlation coefficients suggest that intratester reliability was high for both coronal (.84) and sagittal plane measures (.98), and intertester reliability was high for sagittal plane measures (.89) but moderate for coronal plane meas-

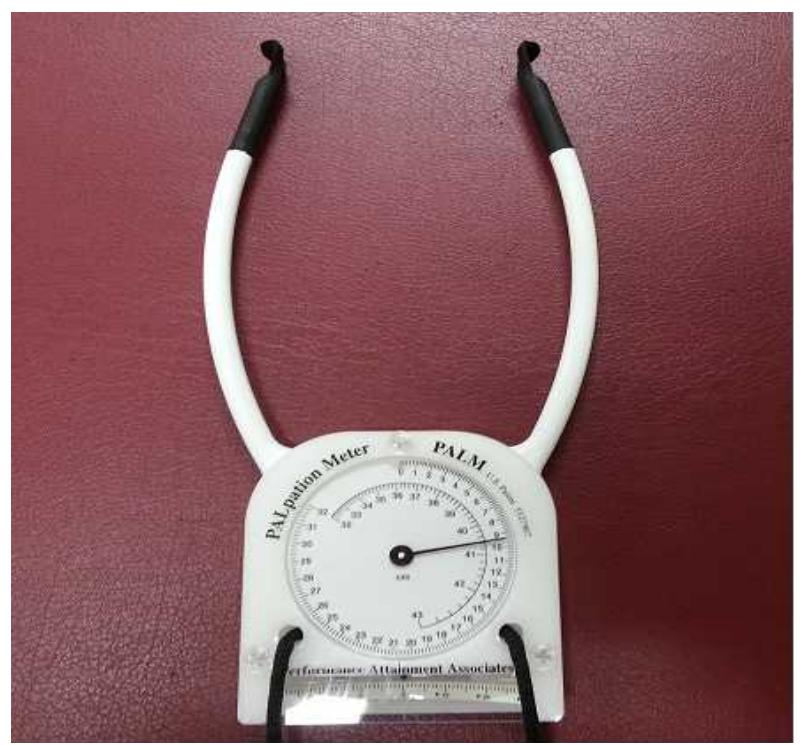

Figure 2. Palpation meter. ures (.65) (Hagins et al, 1998). It consists of an inclinometer and two caliper arms (Lee and Yoo, 2011). The inclinometer, which has a semicircular arc, can measure within the range of $0 \sim 30^{\circ}$ in each direction from the middle at $1^{\circ}$ intervals.

\section{Procedures}

The participants did not wear shoes during the experiment. An experimental work station was created for the typing task. Before subjects sat on the Baltube seat, all air chambers (first to fourth) of the seat were set at $20 \mathrm{mmHg}$. The investigator adjusted the subjects' sitting position to a neutral pelvic position $\left(0^{\circ}\right.$ of coronal and sagittal plane). Subjects were asked to maintain the thoracic spine in a relaxed and comfortable sitting posture and to not change the position of their buttocks. The height of support of Baltube was $43 \mathrm{~cm}$. The hip and knee angles of the subjects were maintained about $90^{\circ}$. Wood box was placed under the feet for adjusting hip and knee $\mathrm{po}^{-}$ sition for short subjects. Subjects were asked to sit with right and left ischium placed on the first and third air chambers and investigator confirmed the ischium position. Participants' elbows were held an angle of $90 \sim 100^{\circ}$ during typing (Gadge and Innes, 2007) and they were asked to maintain their head

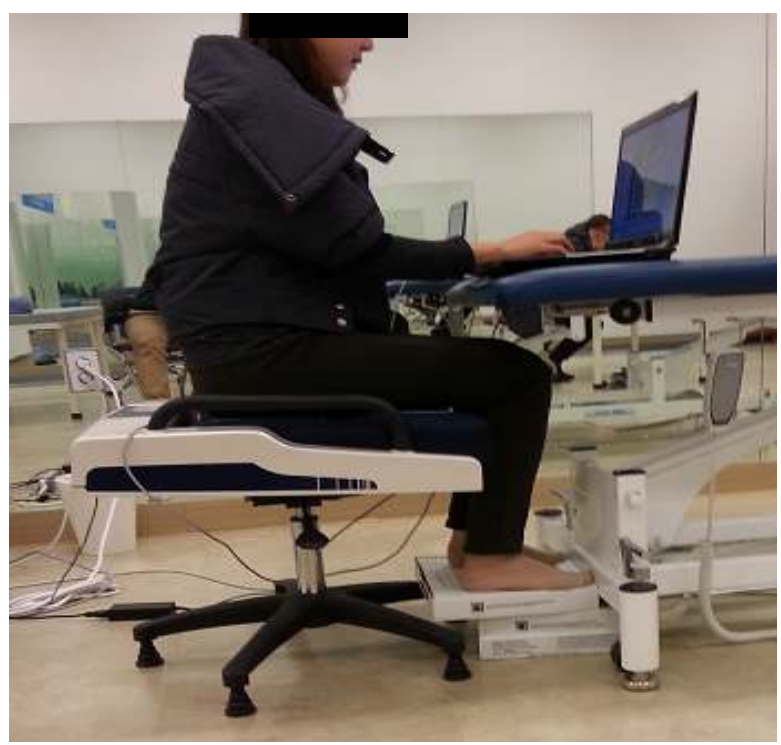

Figure 3. Simulated typing station, with subject sitting on the Baltube. 
directly facing the computer monitor (Figure 3).

The investigator set the height of the typing surface to maintain elbow angle. The distance of the participants from the typing surface was standardized with the edge in line with the radial styloid process and a distance of $30 \mathrm{~cm}$ to the participants' greater trochanter (Gadge and Innes, 2007). A computer with a self-touch keyboard was placed directly in front of the subjects. A typing practice program was used for the task. All subjects typed the same literature (Van Dieën et al, 2001). The literature was "The last leaf" for 30 minutes on each Baltube seat.

\section{Data collection}

\section{Buttock pressure}

Pressure data was collected while the subjects performed a typing task for 30 minutes. The mean pressure value of the initial term was calculated from collected data at the first minute. The mean pressure value of the final term was calculated from collected data pertaining from 29 minutes to the last of the 30 minutes. Pressure asymmetry values were calculated by subtraction the mean pressure of the third chamber (left side) from the mean pressure of the first chamber (right side) in the initial term. Pressure asymmetry value was measured at the initial and final terms. For the unilateral LBP group, pressure change (from the mean pressure of the initial term to the final term) of the symptomatic and asymptomatic sides were calculated.

\section{Pelvic angle}

The pelvic angle was measured after the $30 \mathrm{mi}^{-}$ nutes typing task in the sitting position. The angle of pelvic tilting was measured using the PALM. The investigator palpated the ipsilateral anterior superior iliac spines (ASIS) and the posterior superior iliac spines (PSIS) landmarks palpation through the index fingers. The tips of both index fingers of investigator were inserted in the hole of the PALM. The tips of the PALM were placed on ASIS and PSIS for measuring the angle of pelvic tilting in the sagittal plane. To measure angle of pelvic tilting in the coronal plane, the two tips of the PALM were placed on either side of PSIS. Lateral pelvic tilt (coronal plane) toward the left side indicates minus $(-)$ value and the right side indicates plus $(+)$ value. Anterior pelvic tilt indicates plus $(+)$ value and posterior pelvic tilt indicates minus $(-)$ value.

\section{Statistical analysis}

The SPSS ver. 18.0 software (SPSS Inc., Chicago, IL, USA) was used for statistical analysis. A two-way analysis of variance was used to compare the differences between the initial pressure asymmetry value and the final pressure asymmetry value in subjects with and without unilateral LBP. The significance in terms of intra- and inter-group (between and within) was confirmed. Post hoc analyses were performed to determine pair-wise differences using Bonferroni corrections at the significance level of .012 and a 95\% confidence interval. To compare the change of pressure from the initial term to the final term between the symptomatic and asymptomatic sides in the unilateral LBP group, the paired t-test was applied. The change of pressure was calculated by subtracting the mean of the final term value from the mean of the initial term value. An independent t-test was applied to compare the pelvic tilt angles between the two groups, with the level of statistical significance set at $\mathrm{p}<.05$. The pelvic tilting $\left(^{\circ}\right)$ of the PSIS (coronal plane) and the pelvic tilting ipsilateral ASIS and PSIS (sagittal plane) between the groups with and without unilateral LBP were used in this analysis.

\section{Results}

The initial pressure asymmetry value and final pressure asymmetry value in groups with and without unilateral LBP were viewed in terms of significant differences $(\mathrm{p}<.05)$. The asymmetric pressure 
Table 2. Summary table for two-way ANOVA (mixed design): asymmetry pressure value (mmHg) between the initial and final terms in groups with and without unilateral LBP

\begin{tabular}{cccccc}
\hline \hline Source of variance & $\mathrm{df}^{\mathrm{a}}$ & $\mathrm{SS}^{\mathrm{b}}$ & $\mathrm{MS}^{\mathrm{c}}$ & $\mathrm{F}$ & $\mathrm{p}$ \\
\hline Between subjects & & & & & \\
Group & 1 & 495.42 & 495.42 & 5.39 & \\
Error & 18 & 1655.33 & 91.94 & & \\
Within subjects & & & & & $<.05$ \\
Term & 1 & 640.78 & 640.78 & 63.20 & $<.05$ \\
Group $\times$ Term & 1 & 134.20 & 134.20 & 13.24 & \\
Error & 18 & 182.51 & 10.14 & & \\
\hline
\end{tabular}

${ }^{\mathrm{a}}$ degree of freedom, ${ }^{\mathrm{b}}$ sum of square, ${ }^{\mathrm{c}}$ mean square.

value (differences in pressure between the right and left sides) at the initial and final terms was greater in the unilateral LBP group. Asymmetric pressure value was significantly greater after 30 minutes typing in the unilateral LBP group than in the without unilateral LBP group. Table 2 displays the significance of asymmetry pressure value $(\mathrm{mmHg})$ be $^{-}$ tween the initial and final terms in the groups with and without unilateral LBP. Two-way ANOVA showed a significant main effect both between the groups (with and without unilateral LBP) and within the term (initial and final). Figure 4 shows that the asymmetry pressure value in the initial and final values was $4.52 \mathrm{mmHg}$ and $8.86 \mathrm{mmHg}$, respectively, in the group without unilateral LBP. In the group with unilateral LBP, the asymmetry pressure value in the initial and final values was $7.90 \mathrm{mmHg}$ and $19.56 \mathrm{~mm}$ $\mathrm{Hg}$, respectively. Both the initial and final asymmetry pressure values in the unilateral LBP group were significantly greater than in the without unilateral LBP group. Table 3 displays a comparison of change of buttock pressure between the symptomatic and asymptomatic sides in the unilateral LBP group. Change of buttock of the symptomatic side decreased 7.19 , and change of buttock in the asymptomatic side increased 5.39. There was a significant difference between the symptomatic and asymptomatic sides $(\mathrm{p}<.05)$.

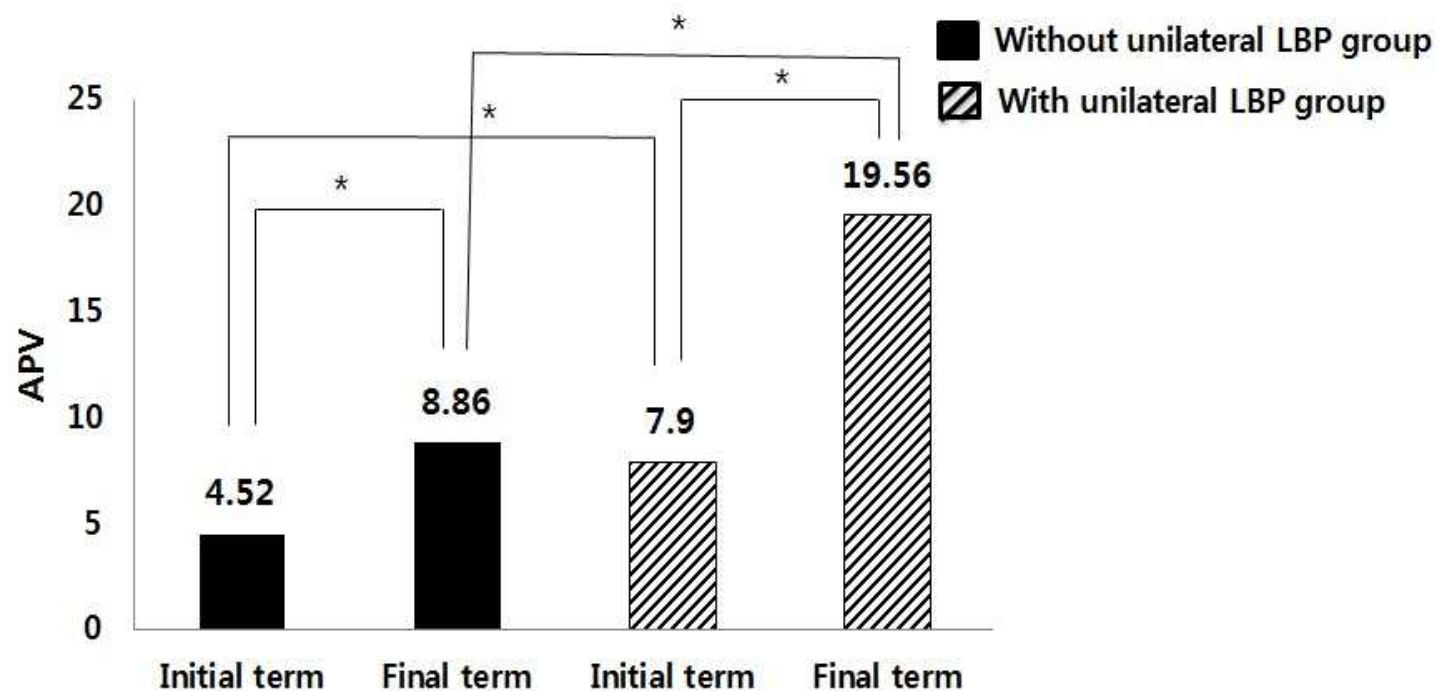

Figure 4. Comparison of asymmetry pressure value (APV) (mmHg) within the initial final terms between groups with and without unilateral LBP [third (left) chamber pressure-first (right) chamber pressure, $\left.{ }^{*} \mathrm{p}<.05\right]$. 
Table 3. Comparison of change of buttock pressure $(\mathrm{mmHg})$ between the symptomatic and asymptomatic sides in the unilateral LBP group

\begin{tabular}{lcccc}
\hline \hline & Symptomatic side & Asymptomatic side & $\mathrm{t}$ & $\mathrm{p}$ \\
\hline Change of buttock pressure $^{\mathrm{a}}$ & $-7.19 \pm 3.84^{\mathrm{b}}$ & $5.39 \pm 3.82$ & -7.66 & $<.05$ \\
\hline${ }^{\mathrm{a}}$ mean pressure of final term-mean & pressure of initial term ${ }^{\mathrm{b}}{ }^{\mathrm{b}}$ mean \pm standard deviation &
\end{tabular}

a mean pressure of final term-mean pressure of initial term, ${ }^{b}$ mean \pm standard deviation.

Table 4. Comparison of the angles of pelvic tilting $\left(^{\circ}\right)$ between the with and without unilateral LBP groups

\begin{tabular}{|c|c|c|c|}
\hline & " Unilateral LBP La $^{\mathrm{a}}$ group & "Without unilateral LBP group & 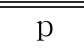 \\
\hline Pelvic angle in coronal plane & $-3.60 \pm 1.08^{b}$ & $-.30 \pm 1.64$ & $<.05$ \\
\hline Pelvic angle in sagittal plane & $-9.80 \pm 5.20$ & $-11.60 \pm 5.85$ & $>.05$ \\
\hline
\end{tabular}

alow back pain, ${ }^{b}$ mean \pm standard deviation.

Table 4 shows differences of pelvic angle in the coronal and sagittal planes. There was a significant difference of pelvic angle on the coronal plane between the groups with and without unilateral LBP. The pelvis was tilted toward the left side by $3.60^{\circ}$ in the unilateral LBP group and $.30^{\circ}$ in the without unilateral LBP group. There was no significant difference in pelvic angle of the sagittal plane between the two groups $(\mathrm{p}>.05)$.

\section{Discussion}

This study was performed to compare the asymmetry of weight bearing and the pelvic tilting angle between subjects with and without unilateral LBP. The result of this study showed that asymmetric pressure values (differences in pressure between the right and left sides) at the initial and final terms and was greater in the unilateral LBP group. Asymmetric pressure value was significantly greater after $30 \mathrm{mi}^{-}$ nutes typing in the unilateral LBP group than in the without unilateral LBP group. In the unilateral LBP group, buttock pressure significantly decreased (7.19 $\mathrm{mmHg}$ ) under the symptomatic side compared to the asymptomatic side. The angle of pelvic tilting in the coronal was significantly greater in the unilateral back pain group the after 30 minutes typing task compared to the without unilateral LBP group. These results support this study's research hypothesis.
Baltube was able to measure the pressure under the right and left buttock separately, therefore asymmetry of buttock pressure between the right and left sides can be measured. Asymmetric sitting posture can induce asymmetric weight bearing and asymmetric pressure between the two sides. In this study, mean buttock pressure was greater under the asymptomatic side during both the initial and final term. Especially, after the 30 minutes typing task, mean buttock pressure decreased more under the symptomatic side. Furthermore the pelvic angle in the coronal plane tilted toward the asymptomatic side after the 30 minutes typing task. These results indicate that the individuals with unilateral LBP shift their weight toward the asymptomatic side. Pelvic lateral tilting induces side-bending in the lumbopelvic segment

Pelvic asymmetry may change biomechanics and increase the strain on bone and soft tissues, resulting in musculoskeletal pain (Egan and $\mathrm{Al}$-Eisa, 1999). If the pelvis is tilted to the left side, right side bending will occur at the lumbopelvic segment. Sharmann (2002) suggests that maintaining asymmetric posture for a long time may cause compression stress on the joint and induce soft tissue adaptation. Prolonged maintain the side-bending to ${ }^{-}$ ward the symptomatic side may cause compression on the symptomatic lumbar facet joint. Aarås et al (1997) reported that LBP patients showed high asymmetric pelvic posture ratios [(ASIS height dif- 
ference/ASIS width $)+($ PSIS height difference/PSIS width)] in lumbar lateral flexion associated with asymmetry. Also a previous study reported that subjects with non specific-LBP appeared to more have asymmetric movement in the low back region and pelvis compared to normal healthy subjects (Al-Eisa et al, 2006). This prolonged asymmetric compression may contribute to osteoarthritic change in the compressed facet joint and evoke unilateral LBP. This asymmetric sitting posture and side-bending of the lumbopelvic segment toward the symptomatic side can be a possible mechanism of unilateral LBP in the sitting position.

The subjects were asked to employ correct and even weight bearing sitting before starting the 30 minutes typing. In addition, the investigator adjusted pelvic position to a neutral position before measurement. But as time passed, asymmetric weight bearing and pelvic tilt progressively increased in the unilateral LBP group. Maintaining asymmetric sitting posture for a long time can induce soft tissue adaptation such as muscle, fascia, ligament, and joint capsule. Weight shift toward the asymptomatic side and lateral tilting of the pelvis toward the asymptomatic side can induce shortening of the soft tissue of the symptomatic side and lengthening of the asymptomatic side. It may cause an imbalance of stiffness in the lumbopelvic segment. It has already been established that greater flexibility toward the symptomatic side compared to the asymptomatic side can be another possible mechanism to explain why the buttock pressure of the symptomatic side progressively $\mathrm{de}^{-}$ creased and the pelvic tilted toward the asymptomatic side as time passed. Also shortened soft tissue in the symptomatic side can induce compression force on facets and disks on the symptomatic side. Adaptive shortening can be another possible mechanism of unilateral LBP. Maintaining a side-bending posture of the lumbar spine can induce unilateral disk compression. If the subjects with unilateral LBP hold asymmetric posture, side-bending of the lumbopelvic segment toward the symptomatic side, the disks in the lumbar can protrude toward the opposite direction.

Park and Yoo (2011) suggest that a posture sensor air seat device gives biofeedback to regular computer workers. The Baltube can modulate seat pressures and apply for exercise LBP and give biofeedback concerning buttock pressure. Further study is needed to discern biofeedback training effect on unilateral LBP and posture correction in individuals with asymmetric sitting posture.

The limitations of this study are as follow, 1) buttock pressure was measured for 30 minutes. Further study is needed to measure the buttock pressure during a longer typing task in sitting position. 2) only young university students were recruited. It is necessary to study people of various ages for generalization. 3) the motion of the lumbopelvic segment was not measured during the $30 \mathrm{mi}^{-}$ nutes typing task. Further study will be needed to measure the motion of the lumbopelvic segment to confirm whether asymmetric pressure induces side-bending of the lumbopelvic segment.

\section{Conclusion}

The result of this study demonstrated that subjects with unilateral LBP sit asymmetrically. Buttock pressure shifts to the asymptomatic side and the pelvis tilts laterally towards the asymptomatic side. This asymmetric sitting posture may contribute to unilateral LBP in the sitting position. Further study is needed to determine whether the correction of sitting posture can decrease unilateral LBP.

\section{References}

Aarås A, Fostervold KI, Ro O, et al. Postural load during VDU work: A comparison between various work postures. Ergonomics. 1997;40( II): 1255-1268. 
Al-Eisa E, Egan D, Deluzio K, et al. Effects of pelvic skeletal asymmetry on trunk movement: Three dimensional analysis in healthy individuals versus patients with mechanical low back pain. Spine (Phila Pa 1976). 2006;31(3): E71-E79.

Al-Eisa E, Egan D, Wassersug R. Fluctuating asymmetry and low back pain. Evol Hum Behav. 2004;25(1):31-37.

Beneck GJ, Kulig K. Multifidus atrophy is localized and bilateral in active persons with chronic unilateral low back pain. Arch Phys Med Rehabil. 2012;93(2):300-306.

Dankaerts W, O'Sullivan P, Burnett A, et al. Differences in sitting postures are associated with non specific chronic low back pain disorders when sub classified. Spine (Phila $\mathrm{Pa}$ 1976). 2006;31(6):698-704.

Egan DA, Cole J, Twomey L. The standing forward flexion test: An inaccurate determinant of $\mathrm{sac}^{-}$ roiliac joint dysfunction. Physiotherapy. 1996; 82(4):236-242.

Egan DA, Al-Eisa E. Pelvic skeletal asymmetry, postural control, and the association with low back pain: A review of the evidence. Crit Rev Phys Rehabil Med. 1999;11:299-338.

Faul F, Erdfelder E, Lang AG, et al. G*power 3: A flexible statistical power analysis program for the social, behavioral, and biomedical sciences. Behav Res Methods. 2007;39(2):175-191.

Gadge K, Innes E. An investigation into the immediate effects on comfort, productivity and posture of the bambach saddle seat and a standard office chair. Work. 2007;29(3):189-203.

Grundy PF, Roberts CJ. Does unequal leg length cause back pain? A case-control study Lancet. 1984;2(8397):256-258.

Hagins M, Brown M, Cook C, et al. Intratester and intertester reliability of the palpation meter (palm) in measuring pelvic position. J Man Manip Ther. 1998;6(3):130-136.

Knutson GA. Incidence of foot rotation, pelvic crest unleveling, and supine leg length alignment asymmetry and their relationship to self-reported back pain. J Manipulative Physiol Ther. 2002;25 (2):110E

Lee JH, Yoo WG. Changes in gluteal pressure and pelvic inclination angles after continuous cross-legged sitting. Work. 2011;40(2):247-252.

Marshall P, Murphy B. Delayed abdominal muscle onsets and self-report measures of pain and disability in chronic low back pain. J Electromyogr Kinesiol. 2010;20(5):833-839.

Maughan EF, Lewis JS. Outcome measures in chronic low back pain. Eur Spine J. 2010;19(9): 1484-1494.

McCaw ST, Bates BT. Biomechanical implications of mild leg length inequality. $\mathrm{Br} \mathrm{J}$ Sports Med. 1991;25(1):10-13.

Nachemson A. Toward a better understanding of back pain: A review of the mechanics of the lumbar disc. Rheumatol Rehabil. 1975;14(3):129143.

Park SY, Yoo YG. Effects of a posture-sensing air seat device (PSASD) on kinematics and trunk muscle activity during continuous computer work. J Physiol Anthropol. 2011;30(4):147-151.

Riegger-Krugh C, Keysor JJ. Skeletal malalignments of the lower quarter: Correlated and compensatory motions and postures. J Orthop Sports Phys Ther. 1996;23(2):164-170.

Sauter SL, Schleifer LM, Knutson SJ. Work posture, workstation design, and musculoskeletal discomfort in a VDT data entry task. Hum Factors. 1991;33(2):151-167.

Sahrmann S. Diagnosis and Treatment of Movement Impairment Syndromes. 1st ed. St Louis, MO, Mosby, 2002:106-108.

Van Dieën, De Looze HP, Hermans V. Effects of dynamic office chairs on trunk kinematics, trunk extensor EMG and spinal shrinkage. Ergonomics. 2001;44(1):739-750.

Vergara M, Page A. Relationship between comfort and back posture and mobility in sitting-posture. 
Appl Ergon. 2002;33(1):1-8.

Watanabe S, Eguchi A, Kobara K, et al. Influence of

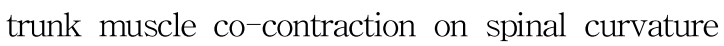
during sitting for desk work. Electromyogr Clin Neurophysiol. 2007;47(6):273-278

Williams MM, Hawley JA, Mckenzie RA et al. A comparison of the effects of two sitting postures on back and referred pain. Spine (Phila $\mathrm{Pa}$
1976). 1991;16(10):1185-1191.

Womersley L, May S. Sitting posture of subjects with postural backache. J Manipulative Physiol Ther. 2006;29(3):213-218.

This article was received January 2, 2014, was reviewed January 2, 2014, and was accepted February 12, 2014. 